\section{UTILIZAÇÃO DA FITOTERAPIA POR UMA PARCELA DE MORADORES DO SETOR BARROS NO MUNICÍPIO DE ARAGUAÍNA - TO}

\section{THE USE OF PHYTOTHERAPY BY PART OF THE RESIDENTS FROM THE BARROS COUNTY OF THE CITY OF ARAGUAÍNA - TO}

Jéssyca Tavares Chaves ${ }^{1}$; Marillya Silva Luz'; Débora Regina Madruga de Vargas ${ }^{2}$

1 Acadêmicas do Curso de Enfermagem; Instituto Tocantinense Presidente Antônio Carlos -

2 Docente Enfermagem. Mestre em Enfermagem. Instituto Tocantinense Presidente Antônio Carlos

\title{
RESUMO
}

A fitoterapia é um método no qual são utilizadas plantas, e seus derivados para o tratamento de enfermidades. A utilização da fitoterapia se dá desde a antiguidade, fazendo parte da evolução humana, tão antiga quanto o próprio homem. $\mathrm{O}$ objetivo deste trabalho foi "revelar a possível utilização das plantas medicinais como forma de tratamento inseridas na cultura popular de um grupo de pessoas de uma zona semirrural de Araguaína - TO". Utilizada metodologia de pesquisa de campo, de caráter qualiquantitativa, exploratória e descritiva. Entrevistados 20 moradores do Setor Barros de Araguaína - TO, com idade superior a 50 anos, onde os dados foram analisados e discutidos baseados no método de Análise de Conteúdo de Bardin. Entre os participantes $80 \%$ destes é do gênero feminino e a média de faixa etária é de 50 a 55 anos. Na pesquisa foram citados 33 diferentes nomes de plantas medicinais, sendo as mais utilizadas: hortelã, cidreira e capim santo, e os principais problemas de saúde citados foram: tosse, febre e gripe. A forma de preparo mais utilizada foram os chás por infusão. A tradição familiar ficou bastante evidente. 0 enfermeiro como profissional que atua diretamente com as classes populares, deve procurar implantar métodos que facilitem a promoção da saúde, através de práticas que estejam ao alcance das pessoas.

\section{PALAVRAS-CHAVE}

Enfermagem.

Fitoterapia.

Plantas Medicinais.

\section{ORRESPON DEN T E}

Débora Regina Madruga de Vargas

Instituto Tocantinense Presidente Antônio Carlos - ITPAC; Av. Filadélfia, 568; Setor Oeste; CEP: 77816-540; Araguaína - TO

E - M A I L

deboramadruga@gmail.com

Recebido: 26/02/2013

Revisado: 04/05/2013

Aprovado: 08/09/2013 


\begin{abstract}
Phytotherapy is a method in which plants, vegetables and derivatives are used for disease treatment. The use of herbal medicine is given since antiquity, being part of human evolution, being as old as man himself. The purpose of the research was "to reveal the possible use of herbal medicines as embedded in popular culture of a certain group of people in a semi-rural area of Araguaína - TO". A field research was the methodology used in qualitative and quantitative nature, exploratory and descriptive. Twenty residents of Barros county of Araguaína - TO were interview, ages 50 years and older, where data was analyzed and discussed based on the Method of Content Analysis of Bandin. Among the $80 \%$ of participants are female and the average age is 50-55 years. Cited in the survey were 33 different names of medicinal plants, of which the most used: mint, lemon balm and lemongrass, and the main health problems mentioned were: cough, fever and flu. The most commonly used form of preparation was infused in teas. The family tradition was very evident. The nurse as a professional, who works directly with the particular groups, should look for methods to facilitate the insertion into promoting their health through practices that are within people's reach.
\end{abstract}

KEYWORDS: Nursing. Phytotherapy. Medicinal Plants.

\section{INTRODUÇÃO}

A utilização das plantas medicinais faz parte da evolução humana sendo tão antiga quanto o próprio homem, pois foi um dos primeiros meios terapêuticos utilizados para se obter a cura de enfermidades, o que revelou seu poder curativo. ${ }^{2}$ Assim, as plantas medicinais representam importante fator para manutenção da saúde das pessoas, uma vez que sua ação terapêutica é comprovada por diversas plantas utilizadas popularmente. ${ }^{12}$

As plantas medicinais "são aquelas que possuem tradição de uso em uma população ou comunidade e são capazes de prevenir, aliviar ou curar enfermidades. Ao serem processadas para obtenção de um medicamento, tem-se como resultado o medicamento fitoterápico" ${ }^{4}$

A utilização da fitoterapia proporciona a interação do ser humano com o meio ambiente, podendo o primeiro ter mais acesso aos recursos que a natureza disponibiliza para ajudar o organismo a normalizar suas funções fisiológicas prejudicadas, restaurar a imunidade enfraquecida, promovendo também a eliminação de substâncias tóxicas e o rejuvenescimento.?

No Brasil, mesmo com o incentivo dos medicamentos industrializados, grande parte da população ainda utilizam meios fitoterápicos como forma de tratamento para aliviar ou curar as enfermidades, principalmente em lugares onde o serviço de saúde é carente, pois a fitoterapia representa parte importante da cultura de uma comunidade, onde também é parte de um conhecimento praticado e transmitido pelas populações ao longo de diversas gerações. ${ }^{2}$

Mesmo havendo os medicamentos sintéticos no mercado, os medicamentos fitoterápicos são muito utilizados pelas pessoas para obter a cura de suas doenças. Porém a utilização indevida e sem o conhecimento dessas plantas é um fator preocupante, pois existem aquelas que contêm toxinas poderosas que podem levar à morte, como também algumas que utilizadas com outros medicamentos podem provocar interações medicamentosas. ${ }^{7}$

Este trabalho buscou esclarecimentos sobre a utilização de plantas medicinais junto à população araguainense, domiciliada em zona semirrural.

Importante ressaltar que a enfermeira como importante membro da equipe multiprofissional poderá prescrever fitoterápicos, porém deverá ter especialização em fitoterapia; qualificada para prestar cuidados de enfermagem e também prescrever medicamentos fitoterápicos, sendo essa atribuição assegurada por lei. Uma vez que essa prática é bastante vivenciada nos postos de saúde, além de preservar a cultura da população presente até os dias de hoje. 


\section{OBJETIVOS}

O objetivo geral deste trabalho é analisar a possível utilização das plantas (fitoterápicos) como medicamentos, inseridas na cultura popular de um grupo de pessoas de uma zona semirrural de Araguaína TO. E tem como objetivos específicos:

» Identificar quais são as plantas medicinais mais utilizadas pelo grupo de pessoas da região abordada; »Verificar o conhecimento que o grupo de pessoas da zona semirrural de Araguaína/TO têm sobre as plantas medicinais em relação às composições, indicações, forma de uso e efeitos das substâncias; "Esclarecer, durante a coleta de dados ao grupo de pessoas da zona semirrural de Araguaína/TO quanto aos possíveis riscos da utilização das plantas medicinais em seu cotidiano, enfatizando a importância do não abandono do tratamento convencional, mas utilizar a fitoterapia como forma de tratamento complementar.

\section{MATERIAIS E MÉTODOS}

Tratou-se de uma pesquisa bibliográfica, de campo, exploratória, descritiva e de ação. A pesquisa de campo consistiu na coleta de dados obtidos através da observação de fatos e fenômenos na íntegra de como ocorrem, onde posteriormente foram analisados e interpretados por meio de etapas, tendo como base um referencial teórico prévio.

A primeira etapa consistiu na pré-análise, onde as informações obtidas foram preparadas para serem analisadas e interpretadas.

Na segunda fase ocorreu a exploração do material, onde os dados foram categorizados de acordo com as características semelhantes apresentadas, conforme os resultados obtidos por meio das entrevistas.

E por fim, a terceira etapa baseou-se na interpretação dos dados obtidos, sendo estes tratados e comparados a literatura sobre o tema proposto, por meio de uma análise crítica e reflexiva.

Com a análise desses dados, pretendeu-se compreender e explicar o problema em questão, que se trata da utilização da fitoterapia em pessoas da zona rural.
A população escolhida para a realização do estudo foram pessoas residentes em área semirrural do município de Araguaína/TO, mais precisamente no Setor Barros, que é composto em seu total por 2.625 pessoas, sendo 1.333 do sexo masculino e 1.292 do sexo feminino, destes 478 estão inseridos na faixa etária acima de 50 anos. (Dados do Sistema de Informação da Atenção Básica (SIAB) fornecidos pela Secretária Municipal de Saúde.)

A escolha por essa comunidade se deu devido ao fato de ser uma região afastada do centro da cidade, sendo considerada semirrural, onde se acredita que a fitoterapia é comumente utilizada para fins terapêuticos, tendo em vista os fatores econômicos e socioculturais.

Participaram da pesquisa os moradores do Setor Barros, onde foram selecionados os indivíduos com idade igual ou superior a 50 anos, independentes das variáveis, sexo, cor, raça ou classe social, bastando apenas residirem no povoado, contabilizando um total de 20 participantes do estudo.

A coleta de dados ocorreu no período compreendido entre a segunda quinzena de agosto e a primeira quinzena de setembro de 2012, após a provação do Comitê de Ética em Pesquisa - FAHESA/ITPAC, sob o parecer consubstanciado ${ }^{\circ} 71778$ e CAAE 04891512.8.0000.0014.

$\mathrm{O}$ instrumento usado para coleta dos dados foi a entrevista semiestruturada. Ou seja, foi criado um questionário composto por um roteiro prévio de perguntas abertas, esse tipo de entrevista é aquela que surge através de questionamentos básicos, estruturados em teorias e hipóteses referentes à pesquisa em questão, que pode oferecer amplo campo de interrogativas posteriores que surgirão à medida que o entrevistado dispõe suas respostas. ${ }^{9}$

Foram feitas aos participantes, perguntas quanto à origem do conhecimento em relação à utilização das plantas medicinais, identificadas apenas pelo nome popular, a relação de cada patologia com as formas de uso ou planta utilizada para cada finalidade, o conhecimento sobre a composição das preparações e se o seu uso já causou algum efeito indesejado. 
As autoras utilizaram um mp3 para gravar as entrevistas com os sujeitos participantes, concedido pelo o Termo de Consentimento Livre e Esclarecido-(TCLE) para que esta fosse gravada. O tempo de entrevista foi variável, pois algumas pessoas, dadas as suas características sempre falam e se expressam mais que outras, todavia, as entrevistas tiveram uma duração de 20 minutos e no máximo de 45 minutos, com cada um dos sujeitos entrevistados.

\section{RESULTADOS E DISCUSSÃO}

\section{Dados Sociodemográficos}

A coleta de dados foi realizada através da entrevista semiestruturada, realizada com 20 moradores do Setor Barros de Araguaína - TO, homens e mulheres escolhidos com idade igual ou superior a 50 anos no período compreendido entre a segunda quinzena de agosto e primeira quinzena de setembro de 2012. Após a coleta de dados estes dados foram analisados e discutidos baseados no método de Análise de Conteúdo proposto por Bardin, que se estrutura basicamente em três etapas: pré-análise, exploração do material e interpretação dos dados obtidos.

A faixa etária predominante foi de pessoas com a idade entre 50 - 55 anos, com um total de 10 (50\%) entrevistados. Na faixa etária compreendida pelas pessoas com idade superior a 66 anos, obtivemos apenas 06 (30\%) entrevistados.

As autoras optaram por realizar a pesquisa com pessoas com idade acima de 50 anos, justamente por suspeitar que o uso da fitoterapia é mais frequente por pessoas dessa faixa etária, e ainda acreditam que quanto maior a idade dos sujeitos maior é o conhecimento da utilização das plantas.

Foi verificado que a distribuição quanto ao gênero dos participantes é predominante o sexo feminino, com 16 (80\%) entrevistados, sendo 04 (20\%) do sexo masculino.

Em relação ao grau de escolaridade dos participantes da pesquisa, verificou-se que 09 entrevistados (45\%), possuem o ensino fundamental incompleto e dentre os não alfabetizados estão 04 entrevistados (20\%). Não houve nenhum participante com ensino superior completo.
As pessoas que possuem o maior conhecimento em relação à utilização das plantas medicinais são pessoas com idade superior a 60 anos e com nível de escolaridade baixo, e a população mais jovem e com maior grau de escolaridade não demonstra interesse em relação à fitoterapia. ${ }^{3}$

Quanto à distribuição dos participantes em relação à renda familiar, foi evidenciado que a maior parte dos entrevistados $13(65 \%)$, possui uma renda que varia de 1 a 3 salários mínimos, e dentre os que possuem 3 a 5 salários mínimos mensais encontramos apenas 02 participantes (10\%). Não foram encontrados participantes com renda superior a cinco salários mínimos, e nenhum participante referiu não possuir rendimentos.

As autoras perceberam que a população estudada, em sua maioria faz o uso da fitoterapia pela falta de outros recursos por serem pessoas de um baixo poder aquisitivo e assim veem a fitoterapia como uma alternativa fácil, mas também por terem essa prática presente em sua família e acreditarem no poder curativo das plantas.

Em relação ao estado civil dos participantes da pesquisa, ficou evidente que a maior proporção é de casados (as) com um total de 17 (85\%) participantes, sendo apenas 03 (15\%) solteiros (as). Nenhum participante referiu ser divorciado (a).

\section{Questionário}

Questão 01 - "Você acredita no poder das plantas medicinais?".

Pela análise feita pelas autoras, houve apenas uma categoria de resposta, denominada de "Crença no poder das plantas medicinais".

As autoras puderam concluir que a fitoterapia é uma prática ainda muito utilizada pela população estudada e que sua crença advém de diversos fatores, como mídia, tradição cultural familiar, crença divina, e experiências adquiridas pela utilização.

Questão 02 - Você utiliza as plantas medicinais com que finalidade?"

Foram determinadas 3 categorias, as quais foram denominadas pelas autoras como: Categoria 01 : Gripe, febre e tosse; Categoria 02: Dores em geral e Categoria 03: Outros fins terapêuticos. 
A gripe é uma doença causada por vários tipos de vírus, e possui alto poder de contaminação. Os primeiros sintomas podem aparecer somente depois de três a quatro dias após a pessoa ser infectada, o que facilita a disseminação do vírus, que é transmitido pela saliva e secreções nasais. ${ }^{16}$ Os principais sintomas são o mal estar, a febre, as dores do corpo e irritação nasal. A febre ou pirexia é caracterizada pela elevação da temperatura corporal do corpo humano ( 36 a 37, $4^{\circ} \mathrm{C}$ ). Pode ser causada por diversos fatores, como: infecções, inflamações, processos malignos dentre outros. ${ }^{16}$

A tosse é um mecanismo fisiológico que atua como um agente que expulsa as secreções respiratórias, sendo considerada, um dos mecanismos de defesa pulmonar. ${ }^{8}$

Ao analisar as falas dos sujeitos as autoras perceberam que entre os entrevistados (as) é forte a presença da utilização das plantas medicinais para o tratamento de sintomas relacionados à dor, e percebeu-se também que essas pessoas tem confiança nessa utilização.

O uso das plantas medicinais com outros fins terapêuticos é considerado uma das mais remotas formas de tratamento, prevenção e cura de doenças usadas pela humanidade, sendo utilizadas muitas vezes como único meio de acesso aos serviços básicos de saúde. ${ }^{8}$

A utilização de plantas medicinais por comunidades para outros fins terapêuticos é o que elas, muitas vezes encontram como forma viável para tratamento de doenças e para manutenção da saúde. ${ }^{14}$

As respostas obtidas foram bem diferentes, alguns dos participantes citaram as plantas que utilizam e para quais patologias fazem esse uso. Os problemas de pressão arterial foram citados, a menopausa, problemas de gastrite, inflamações, dentre outros.

Questão 03 - "Você usou as plantas medicinais por indicação de quem?"

Após a análise das falas dos participantes feita pelas autoras, foram determinadas duas categorias, as quais foram denominadas de Categoria 01: Transmissão de conhecimento entre gerações. Categoria 02: Conhecimento entre pares e de mídia.
Jaconodino et al observaram em um estudo feito que $69 \%$ dos seus sujeitos ao serem indagados sobre o motivo da utilização de terapias alternativas em seu tratamento, responderam que foram influenciados pela indicação de familiares ou amigos. ${ }^{9}$

A medicina popular mantem-se presente e viva no cotidiano das pessoas, onde todas as formas terapêuticas fazem parte do repertório cultural de um conjunto de pessoas que podem ser influenciadas como também influenciar na formação dessa cultura. ${ }^{15}$

Pelos resultados da pesquisa, percebeu-se que a utilização de plantas medicinais ainda é um costume bastante difundido entre pares. Os participantes da pesquisa relataram que essa tradição é repassada por seus familiares, amigos ou conhecidos.

Questão 04 - "Quais são as plantas que você utiliza?" Através da análise feita pelas autoras, surgiu apenas uma categoria, a qual foi denominada de "plantas utilizadas".

Diante da análise da fala dos participantes constatou-se que dentre as plantas citadas pelos participantes, hortelã, cidreira e capim santo, tiveram destaque. Hortelã foi citada 11 vezes, a cidreira foi citada por sete participantes. $\mathrm{O}$ capim santo foi citado seis vezes e tiveram suas indicações comparadas à literatura e visto que elas se confirmam.

Foram muitas as plantas medicinais citadas pelos participantes e as autoras puderam verificar que é forte o conhecimento popular das espécies de plantas medicinais, muitos dos participantes não souberam explicar qual a finalidade dessas plantas, mas ficou evidente a forte presença dessa tradição.

Questão 05 - "Você sabe a maneira correta de utilizar tais plantas?"

Pela a análise feita pelas autoras, surgiu apenas uma categoria, a qual foi denominada de "Método de preparo".

Nesta categoria observou-se que os chás são o método de maior aceitação por parte da população estudada, foram citadas apenas duas maneiras de preparo dos chás, que são a infusão e a maceração.

Os chás podem ser definidos como métodos que se utilizam da água para fazer o preparo adequado das plantas. E possuem quatro maneiras principais de preparo: infusão, decocção, maceração e sucos. ${ }^{5}$ 
A infusão é um método de preparo em que é adicionada água fervente ao recipiente onde estão as ervas medicinais a serem utilizadas, deixando agir por 5 a 10 minutos, para após ingerir o infuso.

Os participantes da pesquisa demonstraram diversas opiniões em relação à maneira que eles julgam correta de fazer o preparo das plantas medicinais. Cada um com sua particularidade demonstrou seu conhecimento e sua tradição em fazer suas preparações. Os chás foram o método predominante e a maneira de preparo desses chás mais utilizada foi o método da infusão, demonstrando o pouco conhecimento que essas pessoas têm das outras formas em que podem ser feitas tais preparações.

Questão 06 - "O que contém cada produto que você utiliza?"

Através da análise feita pelas autoras, surgiu apenas uma categoria, a qual foi denominada de "Água".

Nesta categoria os participantes referiram utilizar na preparação de seus produtos apenas água e às vezes açúcar juntamente com a erva medicinal.

Os chás são considerados a bebida mais ingerida em todo o mundo pela população, perdendo apenas para a água. ${ }^{13} \mathrm{O}$ chá é considerado uma das bebidas

\section{CONFLITOS DE INTERESSE}

Declararam não haver

\section{FONTES DE FINANCIAMENTO}

Nenhuma

\section{REFERÊNCIAS}

1. Badanai JM. Utilização De Plantas Medicinais, Fitoterápicos E Dos Potenciais Riscos De Suas Interações Com Medicamentos Alopáticos, Por Idosos Atendidos Pela Farmácia - Escola - São Caetano Do Sul. Universidade Municipal De São Caetano Do Sul - Uscs. Disponível em:< http://www.uscs.edu.br/ pesquisasacademicas/images/download_inici_cientifica/prof_ celi_e_jaqueline.pdf $>$. Acesso em: 20 de set. 2012 .

2. BADKE, Marcio Rossato, Conhecimento popular sobre o uso de plantas medicinais e o cuidado de enfermagem. 96f. (Dissertação de Mestrado em cuidado educação e trabalho em enfermagem e saúde). Rio Grande do Sul, Universidade Federal de Santa Maria, 2008. Disponível em:<http://www.ufsm.br/ppgenf/ dissertacoes2008/MARCIO_ROSSATO_BADKE.pdf $>$. Acesso em: 20 de mar. 2012.

3. Brasileiro BG et al . Plantas medicinais utilizadas pela população atendida no "Programa de Saúde da Família", Governador Valadares, MG, Brasil. Revista Brasileira de Ciências Farmacêuticas. São Paulo, v. 44, n. 4, Dez. 2008. Disponível em: $<$ http://www.scielo.br/pdf/rbcf/v44n4/v44n4a09.pdf > . Acesso em: 20 de set. 2012 . mais consumidas e também mais antigas do mundo, onde na literatura é referido como uma fonte de moléculas que retardam o envelhecimento celular. ${ }^{11}$

Diante a análise das informações obtidas as autoras verificaram que os participantes utilizam em suas preparações apenas as plantas e não referiram utilizar outros produtos em suas preparações como bebidas alcoólicas, ou outras substâncias químicas.

\section{CONSIDERAÇÕES FINAIS}

Visto que o ser humano busca desde o início de sua existência, alternativas que possam ser a solução de seus males, este trabalho buscou contribuir para que pesquisas em torno da fitoterapia não deixem de ser difundidas e busquem cada vez mais a compreensão dessa modalidade presente na cultura popular.

Profissionais atuantes na enfermagem devem buscar se especializar para transmitirem saberes aos seus pacientes, tendo em vista que este profissional atua diretamente com as classes populares, este deve procurar implantar métodos que facilitem a promoção da saúde, através de práticas que estejam ao alcance dessa população, levando em consideração seus valores e crenças.
4. CONSELHO REGIONAL DE ENFERMAGEM DE SÃO PAULO PARECER COREN-SP GEFIS N 28/2010 Fitoterapia. Legalidade da prescrição de fitoterápicos por Enfermeiro. Prescrição de Correlatos e de Medicamentos por Enfermeiro. Aplicação das Resoluções COFEN 197/1997 e 358/2009.

5. Diniz RC. ABIFISA, Associação Brasileira das Empresas do Setor Fitoterápico, Suplemento Alimentar e Promoção da Saúde. Fitoterapia tem reconhecimento mundial. Folha de Londrina. 2007. Disponível em: < http://www.abifisa.org.br/noticias_ver. asp?news $=1790>$. Acesso em: 22 de set. 2012 .

6. Ferreira VF, Pinto AC. A fitoterapia no mundo atual. Química Nova. São Paulo, v. 33, n. 9, p. 1829, 2010. Disponível em: <http:// dx.doi.org/10.1590/S0100-40422010000900001>. Acesso em: 14 de mar. 2012.

7. França ISX et al. Medicina popular: benefícios e malefícios das plantas medicinais. Revista Brasileira de Enfermagem - REBEn. Brasília, v.61, n.2, pp. 201-208, 2008. Disponível em:< http:// www.scielo.br/pdf/reben/v61n2/a09v61n2.pdf $>$. Acesso em: 21 de mar. 2012 
8. Jacomelli M, Souza R, Pedreira Júnior WL. Abordagem diagnóstica da tosse crônica em pacientes não-tabagistas. Jornal Brasileiro de Pneumologia.N. 504, v. 29, Ed. 6, Nov Dez de 2003. Disponível em: <http://jornaldepneumologia.com.br/PDF/2003_29_6_16_ portugues.pdf $>$. Acesso em: 20 de set. 2012.

9. Jaconodino CB, Amestoy SC, Thofehrn MB. A utilização de terapias alternativas por pacientes em tratamento quimioterápico. Cogitare Enfermagem. [S.1.] v.13, n.1, mar.2008. Disponível em:<http://ojs. c3sl.ufpr.br/ojs2/index.php/cogitare/article/view/11953/8434>. Acesso em: 12 de mar. 2012

10. Martins MCFN, Bógus CM. Considerações sobre a metodologia qualitativa como recurso para o estudo das ações de humanização em saúde. Saúde e Sociedade. São Paulo. v.13, n.3, p.44-57, dez 2004. Disponível em:< http://www.scielo.br/pdf/sausoc/v13n3/06. pdf $>$. Acesso em 20 de mai. 2012.

11. Morais SM et al. Ação antioxidante de chás e condimentos de grande consumo no Brasil. Revista Brasileira de Farmacognosia. João Pessoa, v. 19, n. 1b, Mar. 2009. Disponível em: < http://www. scielo.br/pdf/rbfar/v19n1b/a23v191b.pdf $>$. Acesso em: 21 de set. 2012.

12. OLIVEIRA, Célida Juliana de; ARAUJO, Thelma Leite de. Plantas medicinais: usos e crenças de idosos portadores de hipertensão arterial. Revista Eletrônica de Enfermagem.v. 09, n. 01, p. 93 - 105, 2007. Disponível em:< http://www.fen.ufg.br/revista/v9/n1/pdf/ v9n1a07.pdf >. Acesso em: 02 de abr. 2012.
13. Pegorin F. O branco está na moda. FOLHA DE SÃO PAULO. São Paulo, quinta-feira, 27 de setembro de 2007. Disponível em: < http://wwwl.folha.uol.com.br/fsp/equilibrio/eq2709200709.htm >. Acesso em: 21 de set. 2012.

14. Pinto EPP, Amorozo MCM, Furlan A. Conhecimento popular sobre plantas medicinais em comunidades rurais de mata atlântica Itacaré, BA, Brasil. Acta Bot. Bras., São Paulo, v. 20, n. 4, Dez. 2006 Disponível em: < http://www.scielo.br/pdf/abb/v20n4/01.pdf>. Acesso em: 14 de set. 2012

15. Siqueira KM et al. Crenças populares referentes à saúde: apropriação de saberes sócio-culturais. Texto contexto Enfermagem. Florianópolis, v. 15, n. 1, Mar. 2006. Disponível em $<$ http://www.scielo.br/pdf/tce/v15n1/a08v15n1.pdf $>$. Acesso em: 12 de set. 2012.

16. Spethmann CN. Medicina Alternativa de A a Z (em português). [S.I.]: Natureza, 2004.392 p

17. Veiga Junior VF. Plantas medicinais: Cura Segura?. Química Nova, Vol. 28, No. 3, 519-528, Fev. 2005. Disponível em: < http://www.scielo.br/ pdf/\%0D/qn/v28n3/24145.pdf>. Acesso em: 14 de set. 2012. 